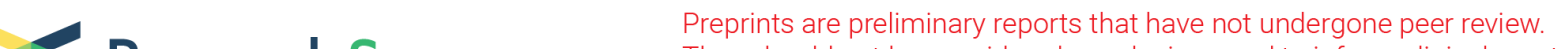

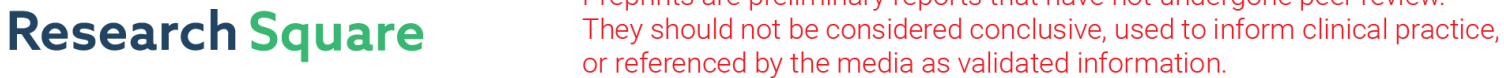

\section{A study of the correlation between M2 macrophages and lymph node metastasis of colorectal carcinoma}

\section{Yanping Wang}

First Affiliated Hospital of Jinzhou Medical University https://orcid.org/0000-0002-1858-1013

\section{Jikun Wang}

First Affiliated Hospital of Jinzhou Medical University

Chunyu Yang

First Affiliated Hospital of Jinzhou Medical University

\section{Yue Wang}

First Affiliated Hospital of Jinzhou Medical University

Jinhao Liu

First Affiliated Hospital of Jinzhou Medical University

\section{Zuoxiu Shi}

First Affiliated Hospital of Jinzhou Medical University

\section{Yanlei Chen}

First Affiliated Hospital of Jinzhou Medical University

\section{Yang Feng}

First Affiliated Hospital of Jinzhou Medical University

\section{Xueqian Ma}

First Affiliated Hospital of Jinzhou Medical University

\section{Shifeng Qiao ( $\square$ shifengqiao2020@163.com )}

The First Affiliated Hospital of Jinzhou Medical University https://orcid.org/0000-0001-9169-7648

\section{Research}

Keywords: M2 macrophages, CD163, colorectal carcinoma, lymph node metastasis, carcinoembryonic antigen

Posted Date: February 18th, 2021

DOI: https://doi.org/10.21203/rs.3.rs-136192/v3

License: (c) (1) This work is licensed under a Creative Commons Attribution 4.0 International License. Read Full License 
Version of Record: A version of this preprint was published at World Journal of Surgical Oncology on March 29th, 2021. See the published version at https://doi.org/10.1186/s12957-021-02195-5. 


\section{Abstract}

Background: Lymph node metastasis is a major prognostic factor of colorectal carcinoma and an important indicator for individualized treatment. M2 macrophages play a key role in carcinogenesis and tumor development, not only enhancing invasiveness, but also promoting lymph node metastasis. The purpose of this study was to investigate the effect of CD163-positive M2 macrophages on lymph node metastasis in colorectal carcinoma.

Methods: Postoperative lymph node tissues were obtained from 120 patients with colorectal carcinoma who underwent radical surgery in the First Affiliated Hospital of Jinzhou Medical University between December 2019 and May 2020. We detected the expression of the CD163 protein in lymph nodes by immunohistochemistry. Furthermore, the relationship between M2 macrophages identified by this marker and lymph node metastasis were analyzed using the independent sample T-test and Chi-square test.

Results: M2 macrophages were increased not only in metastatic lymph nodes, but also in non-metastatic lymph nodes adjacent to the cancer. The M2 macrophage count was higher in patients with macrometastases than in those with micro-metastases.

Conclusions: M2 macrophages represent an important factor for the promotion of lymph node metastasis in colorectal carcinoma, and may be a potential marker for its prediction. This may offer a new target for the comprehensive treatment of colorectal carcinoma.

\section{Introduction}

Colorectal carcinoma (CRC) is one of the most common malignant tumors worldwide, ranking third globally, with an annual incidence of about 1.2 million people. It results in more than 600,000 deaths annually, with a mortality rate increasing year by year[1,2]. Whether or not local lymph node metastasis (LNM) provides important information on tumor stage, clinical treatment and patient prognosis. However, the survival rate of colorectal carcinoma patients with LNM is significantly worse than in patients without LNM [3]. Nonetheless, the mechanisms associated with the origination of LNM remain to be fully elucidated because LNM is an extremely complex process. This involves many immune cells and changes in the expression of many different proteins to enable tumor cells to migrate away from the primary lesion, transit and adhere to, and implant in, the new environment. Compared to normal tissue, lymphatic drainage is increased in tumors. Regional lymph node immune tolerance is a necessary condition for the formation of LNM [4]. The tumor microenvironment (TME ) provides a location for the induction of immune tolerance. The TME is a highly complex network, which in addition to tumor cells, includes a large number of immune cells, comprising macrophages, regulatory $T$ cells, natural killer cells, dendritic cells, $T$ and $B$ lymphocytes as well as non-immune cells such as endothelial cells, cancerassociated fibroblasts and stromal cells. The relative proportions of the cells in the TME are important factors that influence tumor cell invasion and metastasis [5]. Studies have shown that among these individual components, especially M2 macrophages play an important role in promoting tumor growth, 
angiogenesis and lymphangiogenesis, immune tolerance and anti-tumor immunity, and that CD163 is a specific marker for these cells [6, 7].

Many studies have found that the presence of large numbers of M2 macrophages in malignant tumor tissues such as gastric cancer, colorectal carcinoma, breast cancer and cervical cancer is significantly correlated with shortened overall survival [8-11]. However, few studies have reported the relationship between M2 macrophages and LNM. In the present study, the expression of CD163 in lymph node tissue was analyzed to explore the role of M2 macrophages in LNM in colorectal carcinoma, in order to provide more accurate prognostic information, help to identify new molecular therapeutic targets and understand the molecular mechanism of colorectal carcinoma progression.

\section{Materials And Methods}

\section{Patients and specimens}

We collected clinical data and postoperative lymph node specimens of 120 patients with colorectal carcinoma treated at the First Affiliated Hospital of Jinzhou Medical University between December 2019 and May 2020. The clinical characteristics of all patients are shown in Table 1. Inclusion criteria were as follows: 1. Primary colorectal carcinoma; 2 . Diagnosed in our hospital and received surgical treatment for the first time; 3 . Agreed to participate in this study. Exclusion criteria were 1. Received chemotherapy or radiotherapy after diagnosis; 2 . Received targeted immune therapy after diagnosis; 3 . Two or more intestinal malignant tumors or complicated with other systemic malignant tumors. The lymph nodes of patients were divided into five groups as follows: Group A (one normal lymph node was randomly selected from stage I and stage II patients, with a total of 69 cases). Group B (one pathologically-positive node was randomly selected from stage $\nabla$ and $\otimes$ patients, with a total of 51 cases). Group $C$ (one pathologically-negative node was randomly selected from stage $\nabla$ and $\otimes$ patients, with a total of 51 cases). All lymph nodes in group $B$ were divided into two groups according to the size of tumor in the lymph node: group $D(n=32)$ with macro-metastasis $(>2 \mathrm{~mm})$ and group $E(n=19)$ with micrometastasis $(<2 \mathrm{~mm})$ [12]. A total of $171 \mathrm{lymph}$ nodes were examined in this study. Clinical TNM staging was according to the American Joint Committee on Cancer (AJCC) staging standard (8th edition). This study was approved by the Medical Ethics Committee of the First Affiliated Hospital of Jinzhou Medical University, and all patients signed informed consent.

\section{Immunohistochemical staining}

All specimens were fixed in formalin, embedded in paraffin, and cut into sections with a thickness of 4 $\mu \mathrm{m}$. Sections were then deparaffinized with xylene and dehydrated in an ethanol series. Subsequently, antigen retrieval was performed with the ethylenediamine tetra-acetic acid buffer (PH 9.0) at a sub-boiling temperature for $20 \mathrm{~min}$. Tissue slides were incubated with endogenous peroxidase blocker at room temperature for 10 min, blocked with 3\% goat serum (cat. No. KIT-9710; Maixin-Bio, Fuzhou, China) for 30 
min, rinsed with PBS, incubated with ready-to-use mouse anti-human CD163 monoclonal antibody (cat. No. MAB-0206; Maixin-Bio) at room temperature for $60 \mathrm{~min}$, followed by sequential addition of biotinlabeled IgG polymer and Streptavidin peroxidase (cat. No. KIT-9710; Maixin-Bio). Finally, sections were developed using a DAB kit (cat. No. DAB-0031; Maixin-Bio), counterstained with hematoxylin. The density of M2 macrophage infiltration in the lymph nodes was determined by microscopy (Olympus BX43; Japan). All sections were independently evaluated by pathologists (Chun-yu Yang and Yue Wang) using a semi-quantitative scoring system without knowing the patient's clinicopathological data. Immunoreactive score = staining intensity scorexthe score of proportion of cell membrane staining. The staining intensity score was defined as 0 , negative; 1 , weak; 2 , moderate; or 3 , strong, and the score of the proportion of cell membrane staining was defined as $0(<10 \%), 1(11-25 \%), 2(26-50 \%), 3(51-75 \%)$, or $4(76-100 \%)$.

The total score ranged from 0 to 12 . The final immunoreactive score was classed as positive (score $>4$ ) or negative (score <4 ) [13]. Subsequently, five high-power fields (hot spots) with M2 macrophage infiltration were randomly selected under low magnification ( $x 200$ ), the number of M2 macrophages was then counted (per $\mathrm{mm}^{2}$ ) under high magnification ( $\mathrm{x} 400$ ) and the mean number of M2 macrophages was calculated.

\section{Statistical analysis}

SPSS 24.0 software program and GraphPad Prism 8 were used to analyze the data. Measurement data were expressed as mean \pm standard deviation. The independent sample T-test was used to compare the differences in M2 macrophages in lymph node tissues with different clinicopathological parameters, as well as the differences in M2 macrophages between groups. The Chi-square test was used to analyze the association between lymph node metastasis and clinicopathological parameters. Spearman correlation analysis was used for assessing correlations between M2 macrophages and tumor markers. $P<0.05$ was considered to indicate a statistically significant difference.

\section{Results}

\section{Relationships between clinicopathological parameters and lymph node metastasis in colorectal carcinoma patients}

As shown in Table 2, LNM in colorectal carcinoma patients was correlated with the degree of tumor differentiation, depth of invasion, preoperative CEA, CA199 and CA724 levels $(P<0.05)$, but not with gender, age or tumor diameter $(P>0.05)$.

\section{Expression of CD163 protein in lymph node tissues}

Immunohistochemistry showed that the expression of CD163 was characterized by the appearance of yellow or brown granules on the cell membrane of M2 macrophages, whereas cytoplasmic staining was 
slightly weaker. In group A (Figure 1A,1B) and group C (Figure 1C), M2 macrophages mainly infiltrated into the medullary sinus. In group B, M2 macrophages mainly infiltrated into the peritumoral region,but relatively few were to be seen in the intra-tumoral area. (Figure 1D,E).

\section{Mean numbers of M2 macrophages are different in different patient groups}

We found that the mean number of M2 macrophages in group $B(26.8 \pm 7.4)$ was significantly higher than in group $A(14.0 \pm 3.4)$ (Figure. 2A). However, it is not clear whether there is any difference in the number of M2 macrophages in lymph nodes between stage I and $\otimes$ patients and non-metastatic lymph nodes in

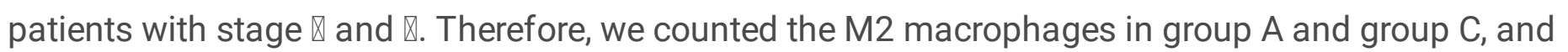
found that the mean number in group $C(17.4 \pm 3.4)$ was significantly higher than in group $A(14.0 \pm 3.4)$ (Fig. 2A). In addition, we also found that the mean number of M2 macrophages in group $D(30.0 \pm 7.0)$ was higher than in group $\mathrm{E}(21.2 \pm 3.9)$ (Figure. 2B).

\section{Relationship between M2 macrophages in lymph node tissue and the clinicopathological features of colorectal carcinoma patients}

In order to identify the characteristics of infiltrating M2 macrophages in lymph nodes, correlations between the mean number of M2 macrophages and patients' clinicopathologic features were sought. It was found that the mean number of M2 macrophages in lymph nodes increased in step with the increase in pathological lymph node $(\mathrm{N})$ categories (Figure. $2 \mathrm{C}$ ). Not only was the mean number of $\mathrm{M} 2$ macrophages in the lymph nodes of patients with pathological tumor $(T)$ categories significantly different (Figure. 2D), but the mean number of M2 macrophages in the lymph nodes of patients with stage III/IV was significantly greater than in patients with stage I/II (Figure. 2E). Moreover, the mean number of M2 macrophages was greater in poorly differentiated metastatic lymph nodes than in moderately and well differentiated lymph nodes (Figure. 2F).

\section{Correlations between the mean number of $\mathrm{M} 2$ macrophages in lymph node tissues and tumor markers}

As shown in Table 3, Spearman analysis was used to seek correlations between the mean number of M2 macrophages and preoperative CEA, CA199, and CA724 levels. These were all found to be positively correlated with the mean number of M2 macrophages $(P<0.05)$.

\section{Discussion}


The results of our studies showed that there was greater M2 macrophage infiltration into metastatic than into non-metastatic lymph nodes, and that more infiltration was seen in patients with macro-metastases than in patients with micro-metastases. It is therefore suggested that M2 macrophages are closely associated with LNM in colorectal carcinoma. In addition, in patients with metastatic lymph nodes, M2 macrophage infiltration into the non-metastatic lymph nodes was also seen, suggesting that the lymph node microenvironment had changed before metastasis, and that $\mathrm{M} 2$ macrophages were involved in that process. Therefore, we speculate that M2 macrophages play an important role in lymph node metastasis of colorectal carcinoma.

The occurrence and development of tumors is closely related to the TME. Macrophages are generally the most abundant component of immune cells in the TME, making up to $50 \%$ of tumor stroma-infiltrating cells [14]. Because macrophages have the characteristics of plasticity and functional diversity, they can be polarized into two types, namely, M1 macrophages (classical activation) and M2 macrophages (alternative activation) depending on changes in the TME[15]. Agents such as lipopolysaccharide (LPS), and cytokines such as Interferon-ץ (IFN- $\gamma$ ) or granulocyte colony-stimulating factor (G-CSF) in the TME influence macrophage differentiation along the M1 pathway. In contrast, macrophages exposed to antiinflammatory cytokines such as IL-4, IL-10, IL-13 or TGF- $\beta$ can be polarized into M2 macrophages. M1 macrophages produce a variety of different pro-inflammatory cytokines, which mainly kill pathogens and tumor cells, and are useful for immune monitoring. M2 macrophages produce less pro-inflammatory cytokines but a large number of anti-inflammatory cytokines, which mainly play an immunosuppressive role and contribute to immune tolerance[16]. Previous studies on colorectal carcinoma indicated that a high M2:M1 ratio was closely related to the enhancement of tumor cell invasion [17]. Our study found that the number of M2 macrophages in lymph nodes was significantly correlated with the depth of tumor invasion, degree of differentiation, degree of lymph node involvement and clinical TNM stage. This indicates that M2 macrophages are involved in the formation of an immunosuppressive environment in lymph nodes and are one of the important factors leading to lymph node metastasis, consistent with previous research results.

Tumor cell metastasis is a stage of deterioration in disease progression and is associated with poor prognosis. Lymphatic metastasis is the most common form of tumor metastasis in various types of malignancies. We speculate that M2 macrophages may facilitate LNM for the following reasons: On the one hand, several studies [18-20] have shown that the number of lymphatic vessels in tumor tissues or metastatic lymph node tissues is significantly higher than that in normal tissues and is related to the presence of M2 macrophages. It has been confirmed that M2 macrophages produce VEGF-C which induces lymphangiogenesis. Tacconi et al. [21]reported that VEGF-C binding to VEGFR3 on lymphatic vessels can inhibit the expression of vascular endothelial cadherin (VE-Cad), resulting in damage to the endothelial barrier of lymphatic vessels around the tumor. This is conducive to the entry of tumor cells into lymphatic vessels. VEGF-C also promotes the proliferation and expansion of lymphatic vessels, which can increase the routes for tumor metastasis to lymph nodes [22]. Therefore, M2 macrophages may reshape the lymphatic network to provide favorable conditions for tumor cell metastasis. 
On the other hand, it may that the presence of a large number of cytokines such as IL-4, EGF, and IL$6[23]$ in the TME. When IL-4 binds to IL-4R, it leads to phosphorylation of JAK-1 and JAK-3, and activates the downstream STAT6 signaling pathway [24]. Choi et al. [25] confirmed that STAT6 phosphorylation increased mRNA expression of M2 macrophage activation markers (FIZZ-1, ARG-1 and CD163), and conversely, that its inhibition reduced the number of M2 macrophages. Yin et al. [26]found that the IL6/JAK/STAT3 signaling pathway was inhibited during M1 macrophage polarization but activated during M2 macrophage polarization. Thus, a new mechanism of IL-6/JAK/STAT3 signaling pathway regulating macrophage polarization was revealed. In addition, Lian et al.[27] noted that colon cancer cells secrete EGF and bind to EGFR on monocytes, which activates the smad-PI3K-Akt-MTOR pathway and promotes monocyte differentiation into M2 macrophages. Therefore, when the above factors are present in normal lymph nodes, they can promote polarization into M2 macrophages. Because these M2 macrophages recruit Tregs into the TME by releasing chemokines (such as CCL 22 and CCL 24)[28], high expression of arginase-1,2 (ARG1,2) and indoleamine-2,3-dioxygenase 1 (IDO1) on the surface of M2 macrophages can greatly deplete arginine and tryptophan from the TME, both of which are indispensable for the metabolism of immune cells, and their depletion leads to T cell and NK cell dysfunction [29-31]. Therefore, M2 macrophages enhance immunosuppression in lymph nodes to create conditions for tumor cell metastasis.

Our study found the mean number of M2 macrophages gradually increased from 9.2 in normal lymph node tissue to 44.4 in metastatic lymph node tissue. We also observed that M2 macrophages mainly infiltrated into the the peritumoral region, but relatively few were to be seen in the intra-tumoral area. Therefore, we speculate that a large number of M2 macrophages infiltrate around tumor cells, leading to the formation of an immunosuppressive TME and the remodeling of the lymphatic network, thus improving the invasion of tumor cells and promoting metastasis. In addition, we also found that preoperative CEA, CA724, and CA199 levels were closely related to lymph node metastasis, and CEA was closely related to the number of $\mathrm{M} 2$ macrophages. Therefore, we speculate that tumor cells may promote the differentiation of M2 macrophages through CEA secretion, thereby promoting lymph node metastasis.

In this study, we comprehensively analyzed the presence of M2 macrophages in lymph node tissues of patients with different stages of colorectal carcinoma, and determined the relationships between them. We found that M2 macrophages were higher not only in the metastatic lymph nodes, but also in the remaining non-metastatic lymph nodes in patients with lymph node metastasis. Therefore, we speculated that M2 macrophages are important factors leading to lymph node metastasis in patients with colorectal carcinoma. Although the specific molecular mechanism whereby M2 macrophages achieve this in colorectal carcinoma is not clear, the results of this study provide a foundation for further research. M2 macrophages have multiple surface markers, including CD68, CD163, and CD206. However, CD 68 is a pan-macrophage marker and cannot distinguish between M1 and M2. In addition, CD206 is expressed not only on macrophages and dendritic cells, but also lymphatic vessels, and hepatic and splenic endothelial cells. CD163 is a type I transmembrane protein with a molecular weight of 130kD. It consists of nine cysteine receptor domains and is a type B scavenger receptor member. It is mainly used for the identification of M2 macrophages, and its role in the polarization of M2 macrophages is the most 
clear $[17,32,33]$. Therefore, CD163 was selected as the marker of choice for M2 macrophages in our study.

In conclusion, M2 macrophages may change the tumor micro-environment and promote lymph node metastasis in colorectal carcinoma. Our findings provide a reference for understanding lymph node metastasis of this cancer and for suggesting treatment targets.

\section{Declarations}

\section{Ethics approval and consent to participate}

The study was conducted in accordance with the Ethics Committee of the First Affiliated Hospital of Jinzhou Medical University and the 1964 Helsinki Declaration. Informed consent was obtained from all participants included in the study.

\section{Consent for publication}

Not applicable

\section{Availability of data and materials}

The analyzed data sets generated during the study are available from the corresponding author on reasonable request. Inquiries for data access may be sent to the following e-mail address: shifengqiao2020@163.com.

\section{Competing interest}

The authors declare that they have no competing interests.

\section{Funding}

Not applicable

\section{Authors' contributions}

Y-PW,C-YY and YW performed the experiments.Y-PW, J-KW and J-HL participated in collecting the data and drafted the manuscript. Z-XS, Y-LC,YF and X-QM contributed to the statistical analysis and manuscript writing. S-FQ conceived the present study and helped revise the manuscript. All authors read and approved the final manuscript.

\section{Acknowledgements}

The authors would like to express their gratitude to EditSprings (https://www.editsprings.com/) for the expert linguistic services provided. 


\section{References}

1. Bray F, Ferlay J, Soerjomataram I, Siegel RL, Torre LA, Jemal A. Global cancer statistics 2018 : GLOBOCAN estimates of incidence and mortality worldwide for 36 cancers in 185 countries. $C A$ Cancer J Clin. 2018;68:394-424.

2. Siegel RL, Miller KD, Fedewa SA, Ahnen DJ, Meester RGS, Barzi A, et al. Colorectal cancer statistics, 2017. CA Cancer J Clin. 2017;67:177-93.

3. O'Connell JB, Maggard MA, Ko CY. Colon cancer survival rates with the new American Joint Committee on Cancer sixth edition staging. J Nat/ Cancer Inst. 2004;96:1420-5.

4. Nathanson SD. Insights into the mechanisms of lymph node metastasis. Cancer. 2003;98:413-23.

5. Quail DF, Joyce JA. Microenvironmental regulation of tumor progression and metastasis. Nat Med. 2013;19:1423-37.

6. Kim KJ, Wen XY, Yang HK, Kim WH, Kang GH. Prognostic Implication of M2 Macrophages Are Determined by the Proportional Balance of Tumor Associated Macrophages and Tumor Infiltrating Lymphocytes in Microsatellite-Unstable Gastric Carcinoma. PLoS One. 2015;10:e0144192.

7. Ding D, Yao Y, Yang C, Zhang S. Identification of mannose receptor and CD163 as novel biomarkers for colorectal cancer. Cancer Biomark. 2018;21:689-700.

8. Park JY, Sung JY, Lee J, Park YK, Kim YW, Kim GY, et al. Polarized CD163+ tumor-associated macrophages are associated with increased angiogenesis and CXCL12 expression in gastric cancer. Clin Res Hepatol Gastroenterol. 2016;40:357-65.

9. Wei C, Yang C, Wang S, Shi D, Zhang C, Lin X, et al. M2 macrophages confer resistance to 5fluorouracil in colorectal cancer through the activation of CCL22/PI3K/AKT signaling. Onco Targets Ther. 2019;12:3051-63.

10. Garvin S, Oda H, Arnesson LG, Lindstrom A, Shabo I. Tumor cell expression of CD163 is associated to postoperative radiotherapy and poor prognosis in patients with breast cancer treated with breastconserving surgery. J Cancer Res Clin Oncol. 2018;144:1253-63.

11. Chen XJ, Han LF, Wu XG, Wei WF, Wu LF, Yi HY, et al. Clinical Significance of CD163+ and CD68+ Tumor-associated Macrophages in High-risk HPV-related Cervical Cancer. J Cancer. 2017;8:3868-75.

12. Hong KD, Um JW, Min BW, Ji WB, Choi JW, Kim YS. Lymph Node Micrometastasis Cannot Be Considered as Positive Lymph Node in Nonmetastatic Colorectal Cancer. Am Surg. 2017;83:127-33.

13. Hu Y, He MY, Zhu LF, Yang CC, Zhou ML, Wang Q, et al. Tumor-associated macrophages correlate with the clinicopathological features and poor outcomes via inducing epithelial to mesenchymal transition in oral squamous cell carcinoma. J Exp Clin Cancer Res. 2016;35:12.

14. Van Overmeire E, Laoui D, Keirsse J, Van Ginderachter JA, Sarukhan A. Mechanisms driving macrophage diversity and specialization in distinct tumor microenvironments and parallelisms with other tissues. Front Immunol. 2014;5:127.

15. Boniakowski AE, Kimball AS, Jacobs BN, Kunkel SL, Gallagher KA. Macrophage-Mediated Inflammation in Normal and Diabetic Wound Healing. J Immunol. 2017;199:17-24. 
16. Grivennikov SI, Greten FR, Karin M. Immunity, inflammation, and cancer. Cell. 2010;140:883-99.

17. Yang C, Wei C, Wang S, Shi D, Zhang C, Lin X, et al. Elevated CD163(+)/CD68(+) Ratio at Tumor Invasive Front is Closely Associated with Aggressive Phenotype and Poor Prognosis in Colorectal Cancer. Int J Biol Sci. 2019;15:984-98.

18. Watanabe M, Tanaka H, Ohira M, Yoshii M, Sakurai K, Toyokawa T, et al. Intranodal lymphangiogenesis precedes development of lymph node metastasis and accelerates progression of gastric cancer. J Gastrointest Surg. 2014;18:481-90.

19. Wu H, Xu JB, He YL, Peng JJ, Zhang XH, Chen CQ, et al. Tumor-associated macrophages promote angiogenesis and lymphangiogenesis of gastric cancer. J Surg Oncol. 2012;106:462-8.

20. Yamagata Y, Tomioka H, Sakamoto K, Sato K, Harada H, Ikeda T, et al. CD163-Positive Macrophages Within the Tumor Stroma Are Associated With Lymphangiogenesis and Lymph Node Metastasis in Oral Squamous Cell Carcinoma. J Oral Maxillofac Surg. 2017;75:2144-53.

21. Tacconi C, Correale C, Gandelli A, Spinelli A, Dejana E, D'Alessio S, et al. Vascular endothelial growth factor $\mathrm{C}$ disrupts the endothelial lymphatic barrier to promote colorectal cancer invasion. Gastroenterology. 2015;148:1438-51 e8.

22. Breslin JW, Gaudreault N, Watson KD, Reynoso R, Yuan SY, Wu MH. Vascular endothelial growth factor-C stimulates the lymphatic pump by a VEGF receptor-3-dependent mechanism. Am J Physiol Heart Circ Physiol. 2007;293:H709-18.

23. Hinshaw DC, Shevde LA. The Tumor Microenvironment Innately Modulates Cancer Progression. Cancer Res. 2019;79:4557-66.

24. Hao J, Hu Y, Li Y, Zhou Q, Lv X. Involvement of JNK signaling in IL4-induced M2 macrophage polarization. Exp Cell Res. 2017;357:155-62.

25. Choi JW, Kwon MJ, Kim IH, Kim YM, Lee MK, Nam TJ. Pyropia yezoensis glycoprotein promotes the M1 to M2 macrophage phenotypic switch via the STAT3 and STAT6 transcription factors. Int $\mathrm{J}$ Mol Med. 2016;38:666-74.

26. Yin Z, Ma T, Lin Y, Lu X, Zhang C, Chen S, et al. IL-6/STAT3 pathway intermediates M1/M2 macrophage polarization during the development of hepatocellular carcinoma. J Cell Biochem. 2018;119:9419-32.

27. Lian G, Chen S, Ouyang M, Li F, Chen L, Yang J. Colon Cancer Cell Secretes EGF to Promote M2 Polarization of TAM Through EGFR/PI3K/AKT/mTOR Pathway. Technol Cancer Res Treat. 2019;18:1533033819849068.

28. Albini A, Bruno A, Noonan DM, Mortara L. Contribution to Tumor Angiogenesis From Innate Immune Cells Within the Tumor Microenvironment: Implications for Immunotherapy. Front Immunol. 2018;9:527.

29. Bantug GR, Galluzzi L, Kroemer G, Hess C. The spectrum of T cell metabolism in health and disease. Nat Rev Immunol. 2018;18:19-34.

30. Heusinkveld M, de Vos van Steenwijk PJ, Goedemans R, Ramwadhdoebe TH, Gorter A, Welters MJ, et al. M2 macrophages induced by prostaglandin E2 and IL-6 from cervical carcinoma are switched to 
activated M1 macrophages by CD4+ Th1 cells. J Immunol. 2011;187:1157-65.

31. Wen Z, Liu H, Li M, Li B, Gao W, Shao Q, et al. Increased metabolites of 5-lipoxygenase from hypoxic ovarian cancer cells promote tumor-associated macrophage infiltration. Oncogene. 2015;34:1241-52.

32. Nielsen MC, Hvidbjerg Gantzel R, Claria J, Trebicka J, Moller HJ, Gronbaek H. Macrophage Activation Markers, CD163 and CD206, in Acute-on-Chronic Liver Failure. Cells. 2020;9.

33. Liu S, Zhang C, Maimela NR, Yang L, Zhang Z, Ping Y, et al. Molecular and clinical characterization of CD163 expression via large-scale analysis in glioma. Oncoimmunology. 2019;8:1601478.

\section{Tables}

Table 1.Patients' characteristics

\begin{tabular}{|c|c|c|c|c|}
\hline & $\begin{array}{c}\text { Group A } \\
(\mathrm{n}=69)(\%)\end{array}$ & Group B or $C(n=51)(\%)$ & $\begin{array}{c}\text { Group D } \\
(\mathrm{n}=32)(\%)\end{array}$ & $\begin{array}{c}\text { Group E } \\
(\mathrm{n}=19)(\%)\end{array}$ \\
\hline Age & & & & \\
\hline Median (range) & $67(49 \sim 86)$ & $63(38 \sim 82)$ & $62(38 \sim 82)$ & $64(50 \sim 81)$ \\
\hline Sex & & & & \\
\hline Male & $40(58)$ & $35(69)$ & $22(69)$ & $13(68)$ \\
\hline Female & $29(42)$ & $16(31)$ & $10(31)$ & $6(32)$ \\
\hline \multicolumn{5}{|c|}{ Anatomic tumor region } \\
\hline Cecum & 1(1) & $3(6)$ & $2(6)$ & $1(5)$ \\
\hline Ascending colon & $9(13)$ & $11(22)$ & $6(19)$ & $5(26)$ \\
\hline Transverse colon & $9(13)$ & $6(12)$ & $3(9)$ & $3(16)$ \\
\hline Descending colon & $6(9)$ & $6(12)$ & $4(13)$ & $2(11)$ \\
\hline Sigmoid & $14(20)$ & $9(18)$ & $6(19)$ & $3(16)$ \\
\hline Rectum & $30(44)$ & $16(30)$ & $11(34)$ & $5(26)$ \\
\hline \multicolumn{5}{|l|}{ T stage (AJCC 8th) } \\
\hline $\mathrm{T} 1$ & $1(1)$ & & & \\
\hline $\mathrm{T} 2$ & $18(27)$ & $3(6)$ & & $3(16)$ \\
\hline T3 & $36(52)$ & $36(71)$ & $21(66)$ & $15(79)$ \\
\hline $\mathrm{T} 4$ & $14(20)$ & $12(23)$ & $11(34)$ & $1(5)$ \\
\hline \multicolumn{5}{|l|}{$\mathrm{N}$ stage (AJCC 8th) } \\
\hline No & $69(100)$ & & & \\
\hline N1 & & $36(71)$ & $19(59)$ & 17(89) \\
\hline $\mathrm{N} 2$ & & $15(29)$ & $13(41)$ & $2(11)$ \\
\hline \multicolumn{5}{|c|}{ Overall stage (AJCC 8th) } \\
\hline प & $19(28)$ & & & \\
\hline$\square \mathrm{A}$ & $36(52)$ & & & \\
\hline aB & $10(14)$ & & & \\
\hline$\square \mathrm{C}$ & $4(6)$ & & & \\
\hline$\square \mathrm{A}$ & & $3(6)$ & & $3(16)$ \\
\hline aB & & $32(62)$ & $19(59)$ & $13(68)$ \\
\hline$\square \mathrm{C}$ & & $9(18)$ & $7(22)$ & $2(11)$ \\
\hline 0 & & $7(14)$ & $6(19)$ & $1(5)$ \\
\hline
\end{tabular}

Table 2. The correlation between the clinicopathologic parameters and lymph node metastasis of colorectal carcinoma 


\begin{tabular}{|c|c|c|c|c|c|}
\hline Clinicopathological parameters & $\begin{array}{c}\text { Number } \\
(\mathrm{n}=120)(\%)\end{array}$ & $\begin{array}{l}\text { Group A } \\
(\mathrm{n}=69)(\%)\end{array}$ & $\begin{array}{l}\text { Group B } \\
(n=51)(\%)\end{array}$ & $\mathrm{C} \square$ & $P$ value \\
\hline Gender & & & & 1.421 & 0.158 \\
\hline Male & $75(63)$ & $40(58)$ & $35(69)$ & & \\
\hline Female & $45(37)$ & $29(42)$ & $16(31)$ & & \\
\hline Age (years) & & & & 0.882 & 0.226 \\
\hline$\geq 65$ & $67(56)$ & $36(52)$ & $31(61)$ & & \\
\hline$<65$ & $53(44)$ & $33(48)$ & 20(39) & & \\
\hline Tumor size $(\mathrm{cm})$ & & & & 1.565 & 0.143 \\
\hline$\geq 5$ & $55(46)$ & $35(51)$ & 20(39) & & \\
\hline$<5$ & $65(54)$ & $34(49)$ & $31(61)$ & & \\
\hline Preoperative CEA (ng/mL) & & & & 7.266 & 0.006 \\
\hline$\geq 5$ & $49(41)$ & $21(30)$ & $28(55)$ & & \\
\hline$<5$ & $71(59)$ & $48(70)$ & $23(45)$ & & \\
\hline Preoperative CA199 (U/mL) & & & & 4.4004 & 0.038 \\
\hline$\geq 37$ & $27(23)$ & $11(16)$ & $16(31)$ & & \\
\hline$<37$ & $93(77)$ & $58(84)$ & $35(69)$ & & \\
\hline Preoperative CA724 (ng/mL) & & & & 28.434 & 0.000 \\
\hline$\geq 6.9$ & $35(29)$ & $7(10)$ & $28(55)$ & & \\
\hline$<6.9$ & $85(71)$ & $62(90)$ & $23(45)$ & & \\
\hline Differentiation degree & & & & 15.408 & 0.000 \\
\hline High/moderate & $88(73)$ & $60(87)$ & $28(55)$ & & \\
\hline Low & $32(27)$ & $9(13)$ & $23(45)$ & & \\
\hline T stage & & & & 4.310 & 0.031 \\
\hline $\mathrm{T} 1+\mathrm{T} 2$ & $22(18)$ & $17(25)$ & $5(10)$ & & \\
\hline $\mathrm{T} 3+\mathrm{T} 4$ & $98(82)$ & $52(75)$ & $46(90)$ & & \\
\hline
\end{tabular}

Table 3. Correlations of the mean number of M2 macrophages with CEA, CA19-9, and CA72-4 levels

\begin{tabular}{lcc}
\hline \multirow{2}{*}{ Tumor markers } & \multicolumn{2}{c}{ the mean number of M2 macrophages } \\
\cline { 2 - 3 } & Spearman correlation coefficient & P value \\
\hline Preoperative CEA & 0.337 & 0.001 \\
Preoperative CA199 & 0.220 & 0.013 \\
Preoperative CA724 & 0.171 & 0.041 \\
\hline
\end{tabular}

\section{Figures}



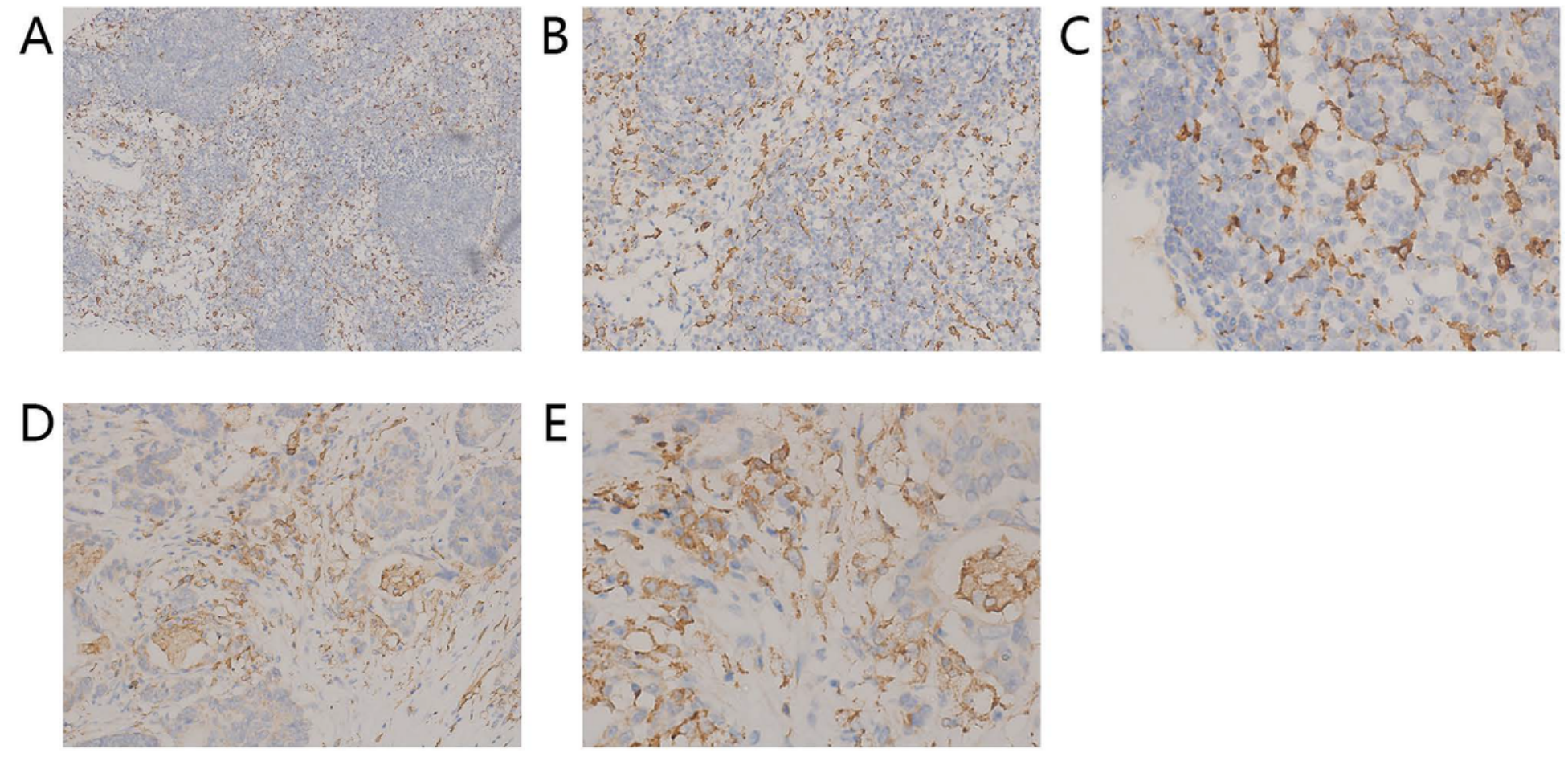

\section{Figure 1}

Expression of M2 macrophages in lymph nodes. Detection of CD163 in lymph nodes of colorectal carcinoma patients by immunohistochemistry. The membrane and cytoplasm of M2 macrophages are stained brown. Microscopic analysis of a typical example of CD163 expression in non-metastatic lymph nodes (A, B, C; magnification x 100, x 200, x 400, respectively). D, E: location of CD163 in metastatic lymph node tissue at a magnification of $x 200$ and $x 400$ respectively. M2 macrophages are seen mainly infiltrating into the tumor stroma. 
A

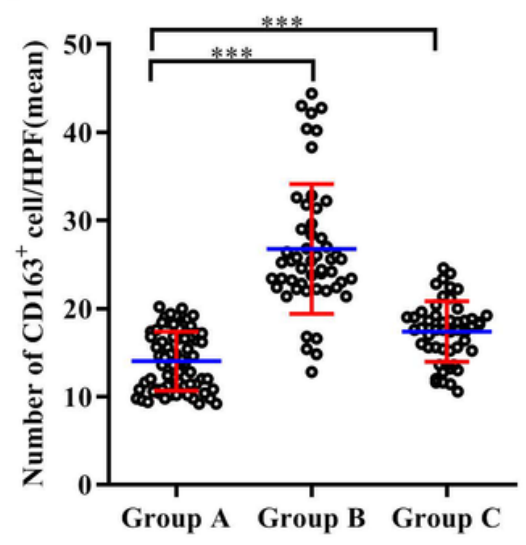

D

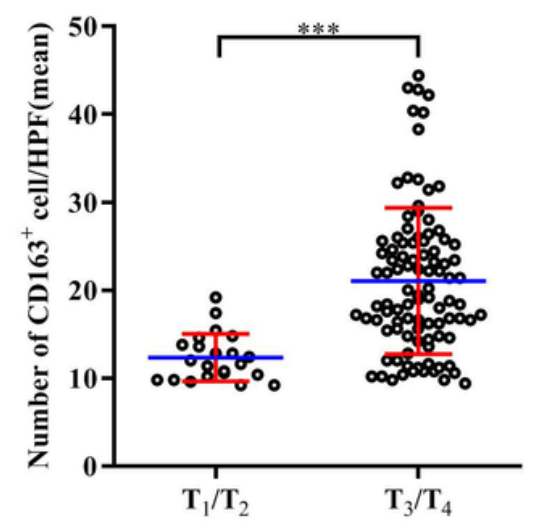

B

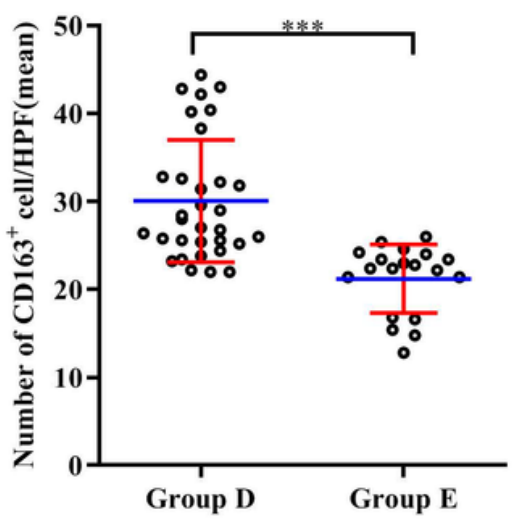

E

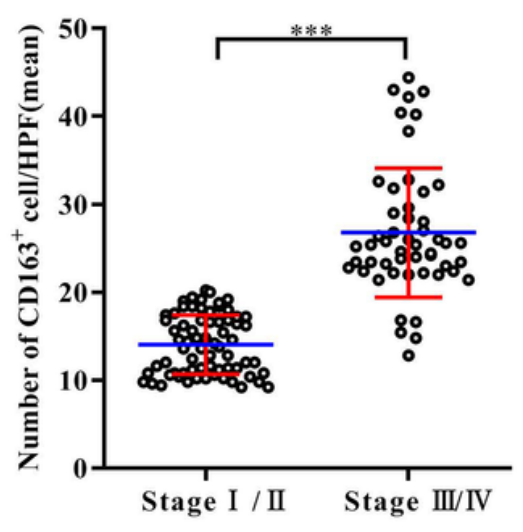

C

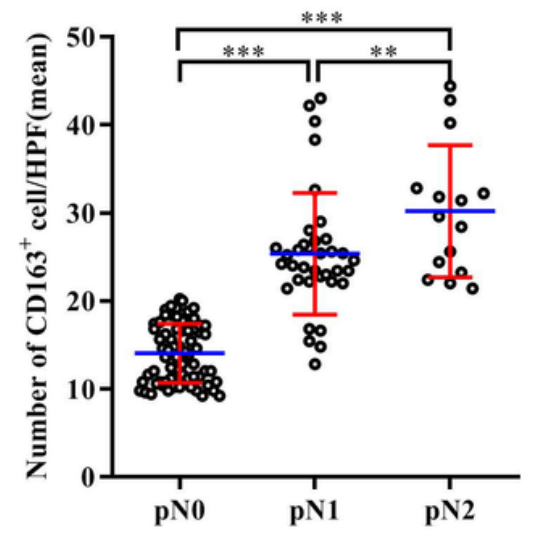

$\mathbf{F}$

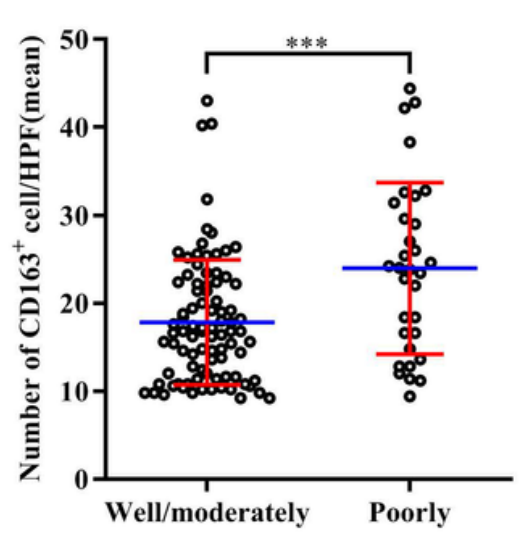

Figure 2

Comparison of the number of M2 macrophages in different groups of lymph nodes and the patients' different clinicopathological features. Comparison of M2 macrophages in lymph nodes of different groups. $A$, the mean number of M2 macrophages in metastatic lymph nodes (Group B) is higher than that in normal lymph nodes (Group $A)\left({ }^{* *} \mathrm{P}<0.0001\right)$, and the mean number of $M 2$ macrophages in nonmetastatic lymph nodes adjacent to cancer (Group C) is higher than that in normal lymph nodes (Group A) $(* \star * P<0.0001) . B$, the mean number of $M 2$ macrophages in macro-metastasis (Group $D)$ is higher than that in micro-metastasis (Group E) $(* \star * P<0.0001)$. Comparison of M2 macrophages among different clinicopathological features. $C$, the number of M2 macrophages is positively correlated with the degree of lymph node metastasis $\left({ }^{\star} * * P<0.0001, * \star P=0.031\right)$. With the increase of pathological tumor $(T)$ categories (D) and clinical TNM stage (E), the mean number of M2 macrophages also increased ( $\left.{ }^{* \star *} P<0.0001\right)$. $F$, the greater the degree of tumor differentiation, the higher the mean number of M2 macrophages $(\star \star * P<0.0001)$. 